\title{
A Facile Pd(0)-Catalyzed Regio- and Stereoselective Diamination of Conjugated Dienes and Trienes
}

\author{
Haifeng Du, Baoguo Zhao, Yian Shi* \\ Department of Chemistry \\ Colorado State University \\ Fort Collins, CO 80523
}

Supporting Information 
Representative diamination procedure (Table 1, entry 1). An NMR tube charged with $\mathrm{Pd}\left(\mathrm{PPh}_{3}\right)_{4}(0.0223 \mathrm{~g}, 0.020 \mathrm{mmol})$ was evacuated and then filled with argon three times. Benzene- $d_{6}(0.6 \mathrm{~mL}$, distilled from sodium) was then added, followed by trans-1,3-pentadiene (5a) $(0.0163 \mathrm{~g}, 0.24 \mathrm{mmol})$ and di-t-butyldiaziridinone $6(0.034 \mathrm{~g}, 0.20 \mathrm{mmol})$. The resulting mixture was immersed into an oil bath $\left(65^{\circ} \mathrm{C}\right)$. Upon completion (as monitored by ${ }^{1} \mathrm{H}$ NMR) (30 $\mathrm{min}$ ), the reaction mixture was purified by flash chromatography (silica gel, hexane:ethyl acetate $=8: 1)$ to give compound $7 \mathbf{a}$ as a colorless oil $(0.045 \mathrm{~g}, 94 \%$ yield $)$.

Bis $\boldsymbol{t}$-butyl deprotection (Scheme 4). To a $5 \mathrm{~mL}$-vial was added compound $\mathbf{7 b}(0.101 \mathrm{~g}, 0.40$ $\mathrm{mmol})$, followed by the addition of $\mathrm{CF}_{3} \mathrm{CO}_{2} \mathrm{H}(0.8 \mathrm{ml})$. Upon stirring at $75-80{ }^{\circ} \mathrm{C}$ for $2 \mathrm{~h}$, the reaction mixture was concentrated and purified by flash chromatography (silica gel, ether to methanol) to give compound $\mathbf{8}$ as a white solid ( $0.055 \mathrm{~g}, 98 \%$ yield).

Mono $\boldsymbol{t}$-butyl deprotection (Scheme 4). To a $5 \mathrm{~mL}$-vial was added compound $7 \mathbf{b}(0.101 \mathrm{~g}, 0.40$ $\mathrm{mmol})$, followed by the addition of $\mathrm{CF}_{3} \mathrm{CO}_{2} \mathrm{H}(0.8 \mathrm{ml})$. Upon stirring at room temperature for 1 $\mathrm{h}$, the reaction mixture was concentrated and purified by flash chromatography (silica gel, ether) to give a white solid containing both $9 \mathbf{a}$ and $9 \mathbf{b}$ (9.3:1) (0.077 g, 98\%).

The above solid $(0.059 \mathrm{~g}, 0.30 \mathrm{mmol})$ was added into a $5 \mathrm{~mL}$-vial, followed by the addition of $\mathrm{CF}_{3} \mathrm{CO}_{2} \mathrm{H}(0.6 \mathrm{ml})$. Upon stirring at $75-80{ }^{\circ} \mathrm{C}$ for $1 \mathrm{~h}$, the reaction mixture was concentrated and purified by flash chromatography (silica gel, ether to methanol) to give compound $\mathbf{8}$ as a white solid ( $0.0403 \mathrm{~g}, 96 \%$ yield).

\section{Table 1, Entry 1}

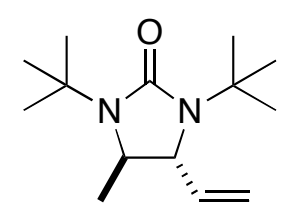

7a, colorless oil; IR (film) $1688 \mathrm{~cm}^{-1}$; ${ }^{1} \mathrm{H}$ NMR $\left(300 \mathrm{MHz}\right.$, benzene- $\left.d_{6}\right) \delta 5.85$ (ddd, $J=17.1$, 9.9, $8.4 \mathrm{~Hz}, 1 \mathrm{H}), 4.95(\mathrm{~d}, J=17.1 \mathrm{~Hz}, 1 \mathrm{H}), 4.86(\mathrm{~d}, J=9.9 \mathrm{~Hz}, 1 \mathrm{H}), 3.25(\mathrm{~d}, J=8.4 \mathrm{~Hz}, 1 \mathrm{H})$, $3.01(\mathrm{q}, J=6.3 \mathrm{~Hz}, 1 \mathrm{H}), 1.44(\mathrm{~s}, 9 \mathrm{H}), 1.37$ (s, 9H), 0,95 (d, $J=6.3 \mathrm{~Hz}, 3 \mathrm{H}) ;{ }^{13} \mathrm{C}$ NMR $(75 \mathrm{MHz}$, benzene- $\left.d_{6}\right) \delta 157.8,140.9,115.4,64.0,55.7,53.3,52.7,29.3,29.2,21.3$; Anal. calcd. for 
$\mathrm{C}_{14} \mathrm{H}_{26} \mathrm{~N}_{2} \mathrm{O}:$ C, 70.54; H, 10.99; N, 11.75; Found: C, 70.66; H, 11.13; N, 12.00; HRMS calcd. for $\mathrm{C}_{14} \mathrm{H}_{27} \mathrm{~N}_{2} \mathrm{O}(\mathrm{M}+1)$ : 239.2123; Found 239.2124.

Table 1, Entry 2

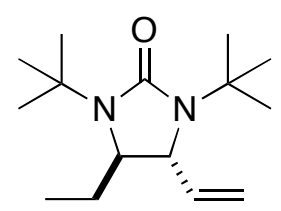

7b, colorless oil; IR (film) $1688 \mathrm{~cm}^{-1} ;{ }^{1} \mathrm{H}$ NMR (300 MHz, benzene- $\left.d_{6}\right) \delta 5.86$ (ddd, $J=17.1$, 9.9, 8.1 Hz, 1H), $5.00(\mathrm{~d}, J=17.1 \mathrm{~Hz}, 1 \mathrm{H}), 4.86(\mathrm{~d}, J=9.9 \mathrm{~Hz}, 1 \mathrm{H}), 3.50(\mathrm{~d}, J=8.1 \mathrm{~Hz}, 1 \mathrm{H})$, $3.03(\mathrm{dd}, J=8.4,2.7 \mathrm{~Hz}, 1 \mathrm{H}), 1.54-1.22(\mathrm{~m}, 2 \mathrm{H}), 1.44$ (s, 9H), 1.39 (s, 9H), 0.72 (t, $J=7.5 \mathrm{~Hz}$, $3 \mathrm{H}) ;{ }^{13} \mathrm{C}$ NMR $\left(75 \mathrm{MHz}\right.$, benzene- $\left.d_{6}\right) \delta 158.2,141.7,115.0,61.2,60.7,53.4,52.8,29.3,29.2$, 27.8, 9.3; Anal. calcd. for $\mathrm{C}_{15} \mathrm{H}_{28} \mathrm{~N}_{2} \mathrm{O}$ : C, 71.38; H, 11.18; N, 11.10; Found: C, 71.50; H, 10.91; N, 11.33; HRMS calcd. for $\mathrm{C}_{15} \mathrm{H}_{29} \mathrm{~N}_{2} \mathrm{O}(\mathrm{M}+1)$ : 253.2280; Found 253.2284.

\section{Table 1, Entry 3}

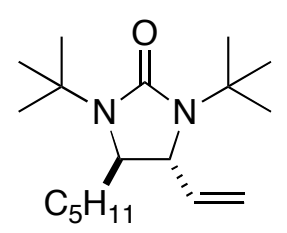

7c, colorless oil; IR (film) $1690 \mathrm{~cm}^{-1}$; ${ }^{1} \mathrm{H}$ NMR (300 MHz, benzene- $\left.d_{6}\right) \delta 5.90$ (ddd, $J=17.1$, 9.9, $8.4 \mathrm{~Hz}, 1 \mathrm{H}), 5.01(\mathrm{~d}, J=17.1 \mathrm{~Hz}, 1 \mathrm{H}), 4.88(\mathrm{~d}, J=9.9 \mathrm{~Hz}, 1 \mathrm{H}), 3.58(\mathrm{~d}, J=8.4 \mathrm{~Hz}, 1 \mathrm{H})$, $3.03(\mathrm{dd}, J=8.7,2.1 \mathrm{~Hz}, 1 \mathrm{H}), 1.66-1.50(\mathrm{~m}, 2 \mathrm{H}), 1.47$ (s, 9H), $1.43(\mathrm{~s}, 9 \mathrm{H}), 1.37-1.10(\mathrm{~m}, 6 \mathrm{H})$, $0.88(\mathrm{t}, J=6.9 \mathrm{~Hz}, 3 \mathrm{H}) ;{ }^{13} \mathrm{C}$ NMR $\left(75 \mathrm{MHz}\right.$, benzene- $\left.d_{6}\right) \delta 158.2,141.6,115.1,61.2,60.1,53.4$, 52.9, 34.9, 32.5, 29.4, 29.3, 25.1, 23.4, 14.6; Anal. calcd. for $\mathrm{C}_{18} \mathrm{H}_{34} \mathrm{~N}_{2} \mathrm{O}: \mathrm{C}, 73.42 ; \mathrm{H}, 11.64 ; \mathrm{N}$, 9.51; Found: C, 73.15; H, 11.79; N, 9.78; HRMS calcd. for $\mathrm{C}_{18} \mathrm{H}_{35} \mathrm{~N}_{2} \mathrm{O}(\mathrm{M}+1)$ : 295.2749; Found 295.2749. 


\section{Table 1, Entry 4}

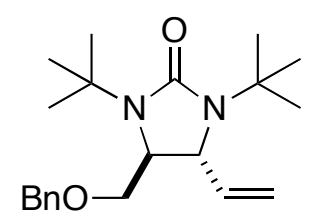

7d, colorless oil; IR (film) $1689 \mathrm{~cm}^{-1}$; ${ }^{1} \mathrm{H}$ NMR (300 MHz, benzene- $\left.d_{6}\right) \delta$ 7.25-7.17 (m, 3H), 7.16-7.05 (m, 2H), $5.90(\mathrm{ddd}, J=18.0,10.2,8.1 \mathrm{~Hz}, 1 \mathrm{H}), 5.16(\mathrm{~d}, J=18.0 \mathrm{~Hz}, 1 \mathrm{H}), 4.92(\mathrm{~d}, J=$ $10.2 \mathrm{~Hz}, 1 \mathrm{H}), 4.32(\mathrm{~d}, J=12.3 \mathrm{~Hz}, 1 \mathrm{H}), 4.20(\mathrm{~d}, J=12.3 \mathrm{~Hz}, 1 \mathrm{H}), 4.04(\mathrm{~d}, J=7.5 \mathrm{~Hz}, 1 \mathrm{H}), 3.36$ (s, 3H), $1.42(\mathrm{~s}, 9 \mathrm{H}), 1.35(\mathrm{~s}, 9 \mathrm{H}) ;{ }^{13} \mathrm{C}$ NMR (75 MHz, benzene- $\left.d_{6}\right) \delta 158.4,140.8,138.9,129.1$, 128.1, 115.9, 73.6, 70.8, 59.4, 59.1, 53.4, 52.9, 29.3, 29.2; Anal. calcd. for $\mathrm{C}_{21} \mathrm{H}_{32} \mathrm{~N}_{2} \mathrm{O}_{2}$ : C, 73.22; H, 9.36; N, 8.13; Found: C, 73.34; H, 9.35; N, 8.24; HRMS calcd. for $\mathrm{C}_{21} \mathrm{H}_{33} \mathrm{~N}_{2} \mathrm{O}_{2}$ $(\mathrm{M}+1)$ : 345.2542; Found 345.2548.

\section{Table 1, Entry 5}

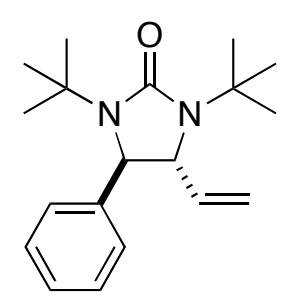

7e, colorless oil; IR (film) $1689 \mathrm{~cm}^{-1}$; ${ }^{1} \mathrm{H}$ NMR (300 MHz, benzene- $\left.d_{6}\right) \delta 7.27$ (d, $J=7.2 \mathrm{~Hz}$, 2H), 7.16-7.02 (m, 3H), $5.92(\mathrm{ddd}, J=17.4,9.9,8.4 \mathrm{~Hz}, 1 \mathrm{H}), 4.91$ (d, $J=17.4 \mathrm{~Hz}, 1 \mathrm{H}), 4.88$ (d, $J=9.9 \mathrm{~Hz}, 1 \mathrm{H}), 4.05(\mathrm{~s}, 1 \mathrm{H}), 3.55(\mathrm{~d}, J=8.4 \mathrm{~Hz}, 1 \mathrm{H}), 1.39(\mathrm{~s}, 9 \mathrm{H}), 1.36(\mathrm{~s}, 9 \mathrm{H}) ;{ }^{13} \mathrm{C} \mathrm{NMR}(75$ MHz, benzene- $\left.d_{6}\right) \delta 159.1,145.0,141.7,129.4,126.4,115.6,65.4,63.8,53.9,53.7,29.2,29.1$; Anal. calcd. for $\mathrm{C}_{19} \mathrm{H}_{28} \mathrm{~N}_{2} \mathrm{O}$ : C, 75.96; H, 9.39; N, 9.32; Found: C, 75.77; H, 9.15; N, 9.35; HRMS calcd. for $\mathrm{C}_{19} \mathrm{H}_{29} \mathrm{~N}_{2} \mathrm{O}(\mathrm{M}+1)$ : 301.2280; Found 301.2284. 
Table 1, Entry 6

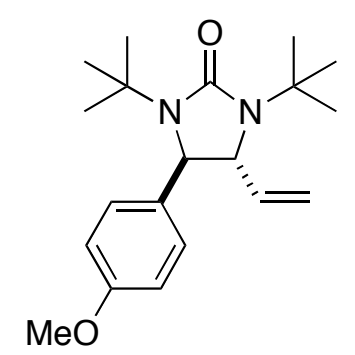

7f, colorless oil; IR (film) 1687, $1610 \mathrm{~cm}^{-1}$; ${ }^{1} \mathrm{H}$ NMR (300 MHz, benzene- $\left.d_{6}\right) \delta$ 7.22-7.17 (m, 2H), 6.72-6.89 (m, 2H), $5.95(\mathrm{ddd}, J=17.4,9.6,8.4 \mathrm{~Hz}, 1 \mathrm{H}), 4.95(\mathrm{~d}, J=17.4 \mathrm{~Hz}, 1 \mathrm{H}), 4.90(\mathrm{~d}$, $J=9.6 \mathrm{~Hz}, 1 \mathrm{H}), 4.05(\mathrm{~s}, 1 \mathrm{H}), 3.58(\mathrm{~d}, J=8.4 \mathrm{~Hz}, 1 \mathrm{H}), 2.29(\mathrm{~s}, 3 \mathrm{H}), 1.44(\mathrm{~s}, 9 \mathrm{H}), 1.40$ (s, 9H); ${ }^{13} \mathrm{C}$ NMR $\left(75 \mathrm{MHz}\right.$, benzene- $\left.d_{6}\right) \delta 160.2,159.1,141.7,136.9,127.6,115.6,114.8,65.7,63.3$, 55.1, 53.8, 53.7, 29.2, 29.1; Anal. calcd. for $\mathrm{C}_{20} \mathrm{H}_{30} \mathrm{~N}_{2} \mathrm{O}_{2}$ : C, 72.69; H, 9.15; N, 8.48; Found: C, 72.34; H, 9.30; N, 8.60; HRMS calcd. for $\mathrm{C}_{20} \mathrm{H}_{31} \mathrm{~N}_{2} \mathrm{O}_{2}(\mathrm{M}+1)$ : 331.2386; Found 331.2386.

\section{Table 1, Entry 7}

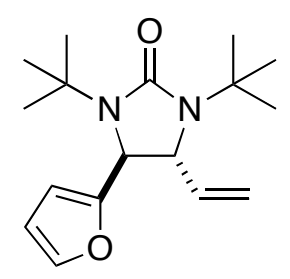

7g, light yellow oil; IR (film) $1693 \mathrm{~cm}^{-1}$; ${ }^{1} \mathrm{H}$ NMR (300 MHz, benzene- $\left.d_{6}\right) \delta 7.00(\mathrm{~d}, J=1.8 \mathrm{~Hz}$ $1 \mathrm{H}), 6.11(\mathrm{~d}, J=3.0 \mathrm{~Hz}, 1 \mathrm{H}), 6.02(\mathrm{dd}, J=3.0,1.8 \mathrm{~Hz}, 1 \mathrm{H}), 5.89(\mathrm{ddd}, J=17.1,9.9,7.8 \mathrm{~Hz}, 1 \mathrm{H})$, $4.97(\mathrm{~d}, J=17.1,1 \mathrm{H}), 4.88(\mathrm{~d}, J=9.9 \mathrm{~Hz}, 1 \mathrm{H}), 4.20(\mathrm{~s}, 1 \mathrm{H}), 3.77(\mathrm{~d}, J=8.1 \mathrm{~Hz}, 1 \mathrm{H}), 1.43(\mathrm{~s}$, 9H), $1.32(\mathrm{~s}, 9 \mathrm{H}) ;{ }^{13} \mathrm{C}$ NMR $\left(75 \mathrm{MHz}\right.$, benzene- $\left.d_{6}\right) \delta 158.5,156.8,142.0,140.6,116.3,111.1$, 106.9, 62.6, 57.7, 53.8, 53.6, 29.1, 28.8; Anal. calcd. for $\mathrm{C}_{17} \mathrm{H}_{26} \mathrm{~N}_{2} \mathrm{O}_{2}$ : C, 70.31; H, 9.02; N, 9.65; Found: C, 70.49; H, 8.88; N, 9.72; HRMS calcd. for $\mathrm{C}_{17} \mathrm{H}_{27} \mathrm{~N}_{2} \mathrm{O}_{2}(\mathrm{M}+1)$ : 291.2073; Found 291.2064. 


\section{Table 1, Entry 8}

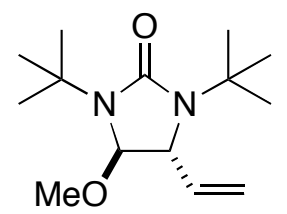

7h, colorless oil; IR (film) $1699 \mathrm{~cm}^{-1} ;{ }^{1} \mathrm{H}$ NMR (300 MHz, benzene- $\left.d_{6}\right) \delta 5.59$ (ddd, $J=17.4$, $10.5,8.1 \mathrm{~Hz}, 1 \mathrm{H}), 5.03(\mathrm{~d}, J=17.4 \mathrm{~Hz}, 1 \mathrm{H}), 4.89(\mathrm{~d}, J=10.5 \mathrm{~Hz}, 1 \mathrm{H}), 4.28(\mathrm{~s}, 1 \mathrm{H}), 3.75(\mathrm{~d}, J=$ 8.1, 1H), $2.93(\mathrm{~s}, 3 \mathrm{H}) 1.48(\mathrm{~s}, 9 \mathrm{H}), 1.40(\mathrm{~s}, 9 \mathrm{H}) ;{ }^{13} \mathrm{C}$ NMR $\left(75 \mathrm{MHz}\right.$, benzene- $\left.d_{6}\right) \delta 158.2,139.8$, 116.6, 90.5, 60.4, 53.8, 53.2, 51.1, 29.1, 28.9; Anal. calcd. for $\mathrm{C}_{14} \mathrm{H}_{26} \mathrm{~N}_{2} \mathrm{O}_{2}$ : C, 66.10; H, 10.30;

N, 11.01; Found: C, 66.05; H, 10.12; N, 11.00; HRMS calcd. for $\mathrm{C}_{14} \mathrm{H}_{27} \mathrm{~N}_{2} \mathrm{O}_{2}(\mathrm{M}+1)$ : 255.2073; Found 255.2074.

\section{Table 1, Entry 9}

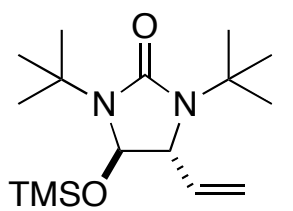

7i, colorless oil; IR (film) $1703 \mathrm{~cm}^{-1}$; ${ }^{1} \mathrm{H}$ NMR (300 MHz, benzene- $\left.d_{6}\right) \delta 5.66$ (ddd, $J=17.4,9.9$, $8.4 \mathrm{~Hz}, 1 \mathrm{H}), 5.08(\mathrm{~d}, J=17.4 \mathrm{~Hz}, 1 \mathrm{H}), 4.90(\mathrm{~d}, J=9.9 \mathrm{~Hz}, 1 \mathrm{H}), 4.76(\mathrm{~s}, 1 \mathrm{H}), 3.73(\mathrm{~d}, J=8.4$, 1H), $1.52(\mathrm{~s}, 9 \mathrm{H}) 1.45(\mathrm{~s}, 9 \mathrm{H}), 0.08(\mathrm{~s}, 9 \mathrm{H}) ;{ }^{13} \mathrm{C}$ NMR (75 MHz, benzene- $\left.d_{6}\right) \delta 158.4,139.2$, 117.0, 84.7, 65.4, 53.8, 53.2, 29.2, 1.0; HRMS calcd. for $\mathrm{C}_{16} \mathrm{H}_{33} \mathrm{~N}_{2} \mathrm{O}_{2} \mathrm{Si}(\mathrm{M}+1)$ : 313.2311; Found 313.2314.

Table 1, Entry 10

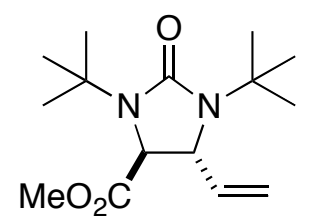

7j, colorless oil; IR (film) 1756, 1737, $1698 \mathrm{~cm}^{-1}$; ${ }^{1} \mathrm{H}$ NMR (300 MHz, benzene-d ${ }_{6}$ ) $\delta 5.78$ (ddd, $J=17.1,9.9,7.8 \mathrm{~Hz}, 1 \mathrm{H}), 5.05(\mathrm{~d}, J=17.1 \mathrm{~Hz}, 1 \mathrm{H}), 4.86(\mathrm{~d}, J=9.9 \mathrm{~Hz}, 1 \mathrm{H}), 3.95(\mathrm{~d}, J=7.8 \mathrm{~Hz}$, 
$1 \mathrm{H}), 3.77(\mathrm{~d}, J=0.9 \mathrm{~Hz}, 1 \mathrm{H}), 3.29(\mathrm{~s}, 3 \mathrm{H}), 1.41(\mathrm{~s}, 9 \mathrm{H}), 1.39(\mathrm{~s}, 9 \mathrm{H}) ;{ }^{13} \mathrm{C}$ NMR $(75 \mathrm{MHz}$, benzene- $\left.d_{6}\right) \delta 173.2,158.4,140.3,116.6,62.0,60.1,53.9,53.7,52.1,29.1,28.7$; Anal. calcd. for $\mathrm{C}_{15} \mathrm{H}_{26} \mathrm{~N}_{2} \mathrm{O}_{3}$ : C, 63.80; H, 9.28; N, 9.92; Found: C, 63.84; H, 9.37; N, 9.67; HRMS calcd. for $\mathrm{C}_{15} \mathrm{H}_{27} \mathrm{~N}_{2} \mathrm{O}_{3}(\mathrm{M}+1)$ : 283.2022; Found 283.2024.

\section{Table 1, Entry 11}

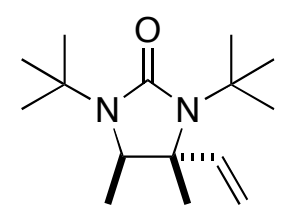

7k, white solid; mp. 39-40 ${ }^{\circ} \mathrm{C}$; IR (film) $1688 \mathrm{~cm}^{-1}$; ${ }^{1} \mathrm{H}$ NMR (300 MHz, benzene- $d_{6}$ ) $\delta 6.22$ (dd, $J=17.4,10.5 \mathrm{~Hz}, 1 \mathrm{H}), 4.89$ (d, $J=17.4 \mathrm{~Hz}, 1 \mathrm{H}), 4.85(\mathrm{~d}, J=10.5 \mathrm{~Hz}, 1 \mathrm{H}), 2.84$ (q, $J=6.0$ $\mathrm{Hz}, 1 \mathrm{H}), 1.51(\mathrm{~s}, 9 \mathrm{H}) 1.39$ (s, 9H), 1.07 (s, 3H), 0.85 (d, $J=6.0 \mathrm{~Hz}, 3 \mathrm{H}) ;{ }^{13} \mathrm{C}$ NMR $(75 \mathrm{MHz}$, benzene- $\left.d_{6}\right) \delta 159.8,144.8,112.8,64.2,60.0,56.4,53.0,30.4,29.1,20.8,16.5$; Anal. calcd. for $\mathrm{C}_{15} \mathrm{H}_{28} \mathrm{~N}_{2} \mathrm{O}$ : C, 71.38; H, 11.18; N, 11.10; Found: C, 71.22; H, 10.91; N, 11.30.

\section{Table 1, Entry 12}

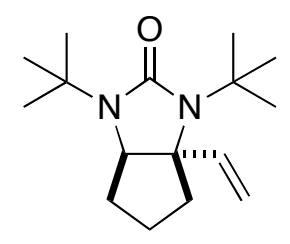

7l, colorless oil; IR (film) $1682 \mathrm{~cm}^{-1}$; ${ }^{1} \mathrm{H}$ NMR $\left(300 \mathrm{MHz}\right.$, benzene- $\left.d_{6}\right) \delta 5.85$ (dd, $J=17.7,10.5$ $\mathrm{Hz}, 1 \mathrm{H}), 4.94(\mathrm{~d}, J=17.7 \mathrm{~Hz}, 1 \mathrm{H}), 4.80(\mathrm{~d}, J=10.5 \mathrm{~Hz}, 1 \mathrm{H}), 3.16(\mathrm{t}, J=7.2 \mathrm{~Hz}, 1 \mathrm{H}), 1.97-1.87$ (m, 1H), 1.75-1.60 (m, 2H), 1.53 (s, 9H), 1.50-1.20 (m, 2H), 1.39 (s, 9H), 1.18-0.95 (m, 1H); ${ }^{13} \mathrm{C}$ NMR (100 MHz, benzene- $\left.d_{6}\right) \delta 158.9,146.3,111.9,69.7,67.3,55.1,53.4,39.6,37.8,30.1$, 29.2, 24.8; HRMS calcd. for $\mathrm{C}_{16} \mathrm{H}_{29} \mathrm{~N}_{2} \mathrm{O}(\mathrm{M}+1)$ : 265.2280; Found 265.2281. 


\section{Table 1, Entry 13}

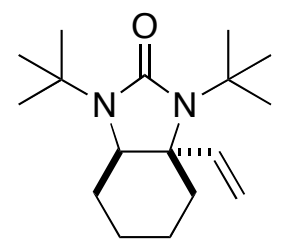

7m, colorless oil; IR (film) $1687 \mathrm{~cm}^{-1} ;{ }^{1} \mathrm{H}$ NMR (300 MHz, benzene- $\left.d_{6}\right) \delta 6.19(\mathrm{dd}, J=18.0$, $11.1 \mathrm{~Hz}, 1 \mathrm{H}), 4.95(\mathrm{~d}, J=18.0 \mathrm{~Hz}, 1 \mathrm{H}), 4.91(\mathrm{~d}, J=11.1 \mathrm{~Hz}, 1 \mathrm{H}), 2.80(\mathrm{dd}, J=9.3,5.1 \mathrm{~Hz}, 1 \mathrm{H})$, 2.10-1.95 (m, 1H), $1.53(\mathrm{~s}, 9 \mathrm{H}), 1.51-1.21(\mathrm{~m}, 6 \mathrm{H}), 1.38(\mathrm{~s}, 9 \mathrm{H}), 1.00-0.84(\mathrm{~m}, 1 \mathrm{H})$; ${ }^{13} \mathrm{C} \mathrm{NMR}$ $\left(75 \mathrm{MHz}\right.$, benzene- $\left.d_{6}\right) \delta 160.0,143.9,113.1,64.3,60.3,56.7,52.7,30.9,30.2,29.5,29.1,21.9$, 20.4; HRMS calcd. for $\mathrm{C}_{17} \mathrm{H}_{31} \mathrm{~N}_{2} \mathrm{O}(\mathrm{M}+1)$ : 279.2436; Found 279.2444.

\section{Table 1, Entry 14}

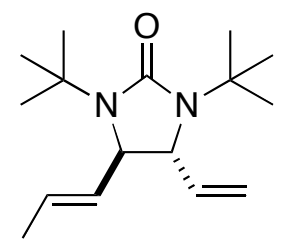

7n, white solid; mp. $63-64{ }^{\circ} \mathrm{C}$; IR (film) $1692 \mathrm{~cm}^{-1}$; ${ }^{1} \mathrm{H}$ NMR (300 MHz, benzene- $\left.d_{6}\right) \delta 5.94$ (ddd, $J=17.4,10.2,8.4 \mathrm{~Hz}, 1 \mathrm{H}), 5.70-5.60$ (m, 1H), 5.40-5.28 (m, 1H), 4.99 (d, $J=17.4 \mathrm{~Hz}$, $1 \mathrm{H}), 4.88(\mathrm{~d}, J=10.2 \mathrm{~Hz}, 1 \mathrm{H}), 3.52(\mathrm{~d}, J=7.5 \mathrm{~Hz}, 1 \mathrm{H}), 3.50(\mathrm{~d}, J=8.1 \mathrm{~Hz}, 1 \mathrm{H}), 1.47-1.42(\mathrm{~m}$, $21 \mathrm{H}) ;{ }^{13} \mathrm{C}$ NMR $\left(75 \mathrm{MHz}\right.$, benzene- $\left.d_{6}\right) \delta 158.4,140.7,133.3,127.1,115.8,63.8,63.0,53.6,53.5$, 29.2, 17.9; HRMS calcd. for $\mathrm{C}_{16} \mathrm{H}_{29} \mathrm{~N}_{2} \mathrm{O}(\mathrm{M}+1)$ : 265.2280; Found 265.2285.

\section{Table 1, Entry 15}

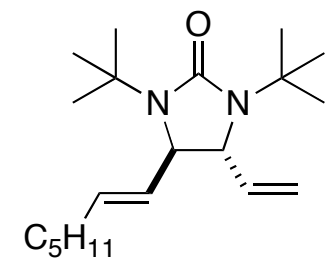

7o, colorless oil; IR (film) $1692 \mathrm{~cm}^{-1} ;{ }^{1} \mathrm{H}$ NMR (300 MHz, benzene- $\left.d_{6}\right) \delta 5.96$ (ddd, $J=17.1$, 9.9, 8.4 Hz, 1H), $5.68(\mathrm{dd}, J=15.3,8.4 \mathrm{~Hz}, 1 \mathrm{H}), 5.43(\mathrm{dt}, J=15.3,6.9 \mathrm{~Hz}, 1 \mathrm{H}), 5.01(\mathrm{~d}, J=17.1$ 
$\mathrm{Hz}, 1 \mathrm{H}), 4.90(\mathrm{~d}, J=9.9 \mathrm{~Hz}, 1 \mathrm{H}), 3.57(\mathrm{~d}, J=7.5 \mathrm{~Hz}, 1 \mathrm{H}), 3.55(\mathrm{~d}, J=7.8 \mathrm{~Hz}, 1 \mathrm{H}), 1.89(\mathrm{dt}, J=$ 6.9, $6.6 \mathrm{~Hz}, 2 \mathrm{H}), 1.48(\mathrm{~s}, 9 \mathrm{H}), 1.46(\mathrm{~s}, 9 \mathrm{H}), 1.34-1.14(\mathrm{~m}, 6 \mathrm{H}), 0.87(\mathrm{t}, J=6.6 \mathrm{~Hz}, 3 \mathrm{H}) ;{ }^{13} \mathrm{C}$ NMR (75 MHz, benzene- $\left.d_{6}\right) \delta 158.4,140.7,132.8,132.1,115.8,63.9,63.0,53.6,53.5,32.7$, 32.0, 29,5, 29.2, 23.1, 14.6; HRMS calcd. for $\mathrm{C}_{20} \mathrm{H}_{37} \mathrm{~N}_{2} \mathrm{O}(\mathrm{M}+1)$ : 321.2906; Found 321.2904.

\section{Table 1, Entry 16}

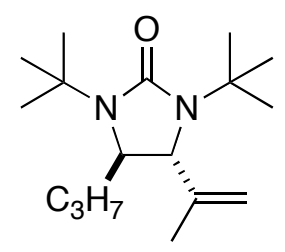

7p, colorless oil; IR (film) $1687 \mathrm{~cm}^{-1}$; ${ }^{1} \mathrm{H}$ NMR (300 MHz, benzene- $\left.d_{6}\right) \delta 4.90(\mathrm{~s}, 1 \mathrm{H}), 4.72-$ $4.68(\mathrm{~m}, 1 \mathrm{H}), 3.52(\mathrm{~s}, 1 \mathrm{H}), 2.99(\mathrm{dd}, J=7.8,2.1 \mathrm{~Hz}, 1 \mathrm{H}), 1.64(\mathrm{~s}, 3 \mathrm{H}), 1.44(\mathrm{~s}, 9 \mathrm{H}), 1.40(\mathrm{~s}, 9 \mathrm{H})$, $1.50-1.23(\mathrm{~m}, 3 \mathrm{H}), 1.20-1.06(\mathrm{~m}, 1 \mathrm{H}), 0.82(\mathrm{t}, J=7.2 \mathrm{~Hz}, 3 \mathrm{H}) ;{ }^{13} \mathrm{C}$ NMR $(100 \mathrm{MHz}$, benzene$\left.d_{6}\right) \delta 158.5,148.5,111.8,63.1,59.1,53.6,52.8,38.7,29.4,29.1,18.4,18.2,14.6$; Anal. calcd. for $\mathrm{C}_{17} \mathrm{H}_{32} \mathrm{~N}_{2} \mathrm{O}$ : C, 72.81; H, 11.50; N, 9.99; Found: C, 73.06; H, 11.69; N, 10.00; HRMS calcd. for $\mathrm{C}_{17} \mathrm{H}_{33} \mathrm{~N}_{2} \mathrm{O}(\mathrm{M}+1)$ : 281.2593; Found 281.2589.

\section{Table 1, Entry 17}

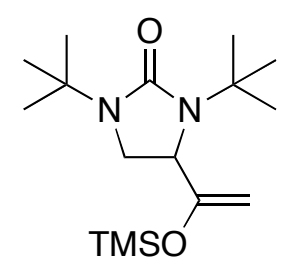

7q, colorless oil; IR (film) $1695 \mathrm{~cm}^{-1}$; ${ }^{1} \mathrm{H}$ NMR $\left(300 \mathrm{MHz}\right.$, benzene- $\left.d_{6}\right) \delta 4.33(\mathrm{~d}, J=1.8, \mathrm{~Hz}$, $1 \mathrm{H}), 4.07(\mathrm{~d}, J=1.8 \mathrm{~Hz}, 1 \mathrm{H}), 3.71(\mathrm{dd}, J=9.3,3.0 \mathrm{~Hz}, 1 \mathrm{H}), 3.10(\mathrm{dd}, J=9.3,8.4 \mathrm{~Hz}, 1 \mathrm{H}), 2.99$ (dd, $J=8.4,3.0 \mathrm{~Hz}, 1 \mathrm{H}), 1.51(\mathrm{~s}, 9 \mathrm{H}) 1.34(\mathrm{~s}, 9 \mathrm{H}), 0.15(\mathrm{~s}, 9 \mathrm{H}) ;{ }^{13} \mathrm{C}$ NMR $(75 \mathrm{MHz}$, benzene$\left.d_{6}\right) \delta$ 161.1, 160.8, 89.3, 56.1, 54.2, 53.0, 47.5, 29.0, 27.8, 0.3; Anal. calcd. for $\mathrm{C}_{16} \mathrm{H}_{32} \mathrm{~N}_{2} \mathrm{O}_{2} \mathrm{Si}: \mathrm{C}$, 61.49; H, 10.32; N, 8.96; Found: C, 61.70; H, 10.14; N, 9.16; HRMS calcd. for $\mathrm{C}_{16} \mathrm{H}_{33} \mathrm{~N}_{2} \mathrm{O}_{2} \mathrm{Si}$ (M+1): 313.2311; Found 313.2314. 


\section{Scheme 4}

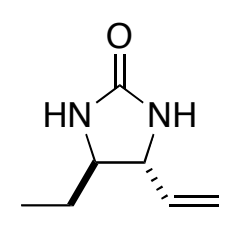

8, white solid; mp. $177-178{ }^{\circ} \mathrm{C}$; IR (film) 3206, $1704 \mathrm{~cm}^{-1} ;{ }^{1} \mathrm{H}$ NMR (300 MHz, $\left.\mathrm{CDCl}_{3}\right) \delta 5.85$ (ddd, $J=17.1,9.9,7.5 \mathrm{~Hz}, 1 \mathrm{H}), 5.27(\mathrm{~d}, J=17.1 \mathrm{~Hz}, 1 \mathrm{H}), 5.18(\mathrm{~d}, J=9.9 \mathrm{~Hz}, 1 \mathrm{H}), 4.95$ (bs, $1 \mathrm{H})$, $4.73(\mathrm{bs}, 1 \mathrm{H}), 3.86(\mathrm{dd}, J=7.5,6.9 \mathrm{~Hz}, 1 \mathrm{H}), 3.37$ (td, $J=7.2,6.9 \mathrm{~Hz}, 1 \mathrm{H}), 1.73-1.48(\mathrm{~m}, 2 \mathrm{H})$, $0.96(\mathrm{t}, J=7.5 \mathrm{~Hz}, 3 \mathrm{H}) ;{ }^{13} \mathrm{C} \mathrm{NMR}\left(75 \mathrm{MHz}, \mathrm{CDCl}_{3}\right) \delta 163.5,137.8,117.1,61.6,60.6,27.9,10.2$; Anal. calcd. for $\mathrm{C}_{7} \mathrm{H}_{12} \mathrm{~N}_{2} \mathrm{O}$ : C, 59.98; H, 8.63; N, 19.98; Found: C, 59.78; H, 8.43; N, 20.10.

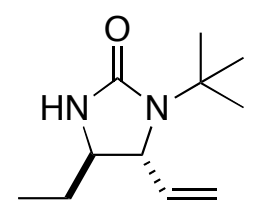

9a, white solid; mp. $58-59{ }^{\circ} \mathrm{C}$; IR (film) $3225,1693 \mathrm{~cm}^{-1}$; ${ }^{1} \mathrm{H}$ NMR (300 MHz, acetontrile- $\left.d_{3}\right) \delta$ 6.01(ddd, $J=17.1,10.2,8.1 \mathrm{~Hz}, 1 \mathrm{H}), 5.26(\mathrm{~d}, J=17.1 \mathrm{~Hz}, 1 \mathrm{H}), 5.15(\mathrm{~d}, J=10.2 \mathrm{~Hz}, 1 \mathrm{H}), 4.04$ $(\mathrm{dd}, J=8.1,2.4 \mathrm{~Hz}, 1 \mathrm{H}), 3.09(\mathrm{dd}, J=6.3,2.4 \mathrm{~Hz}, 1 \mathrm{H}), 1.59-1.40(\mathrm{~m}, 2 \mathrm{H}), 1.36(\mathrm{~s}, 9 \mathrm{H}), 0.90(\mathrm{t}$, $J=7.5 \mathrm{~Hz}, 3 \mathrm{H}) ;{ }^{13} \mathrm{C} \mathrm{NMR}\left(75 \mathrm{MHz}\right.$, acetontrile- $\left.d_{3}\right) \delta 163.4,141.1,117.1,65.1,56.0,55.8,29.2$, 28.8, 9.6; HRMS calcd. for $\mathrm{C}_{11} \mathrm{H}_{21} \mathrm{~N}_{2} \mathrm{O}(\mathrm{M}+1)$ : 197.1654; Found 197.1650.

\section{NOE studies to determine stereochemistry}

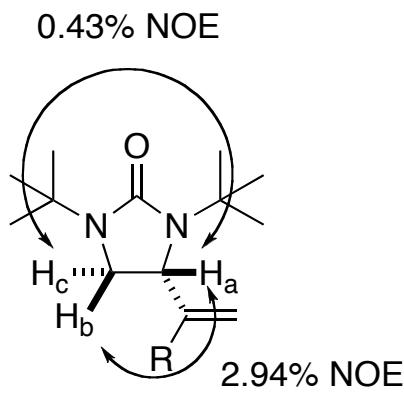

7q, $R=$ OTMS

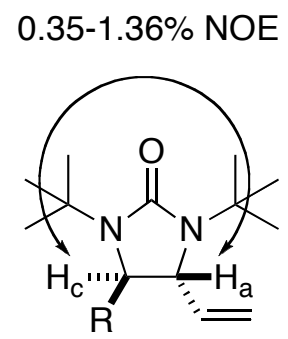

7b, g, h, p 
X-ray structure for $7 \mathbf{n}$
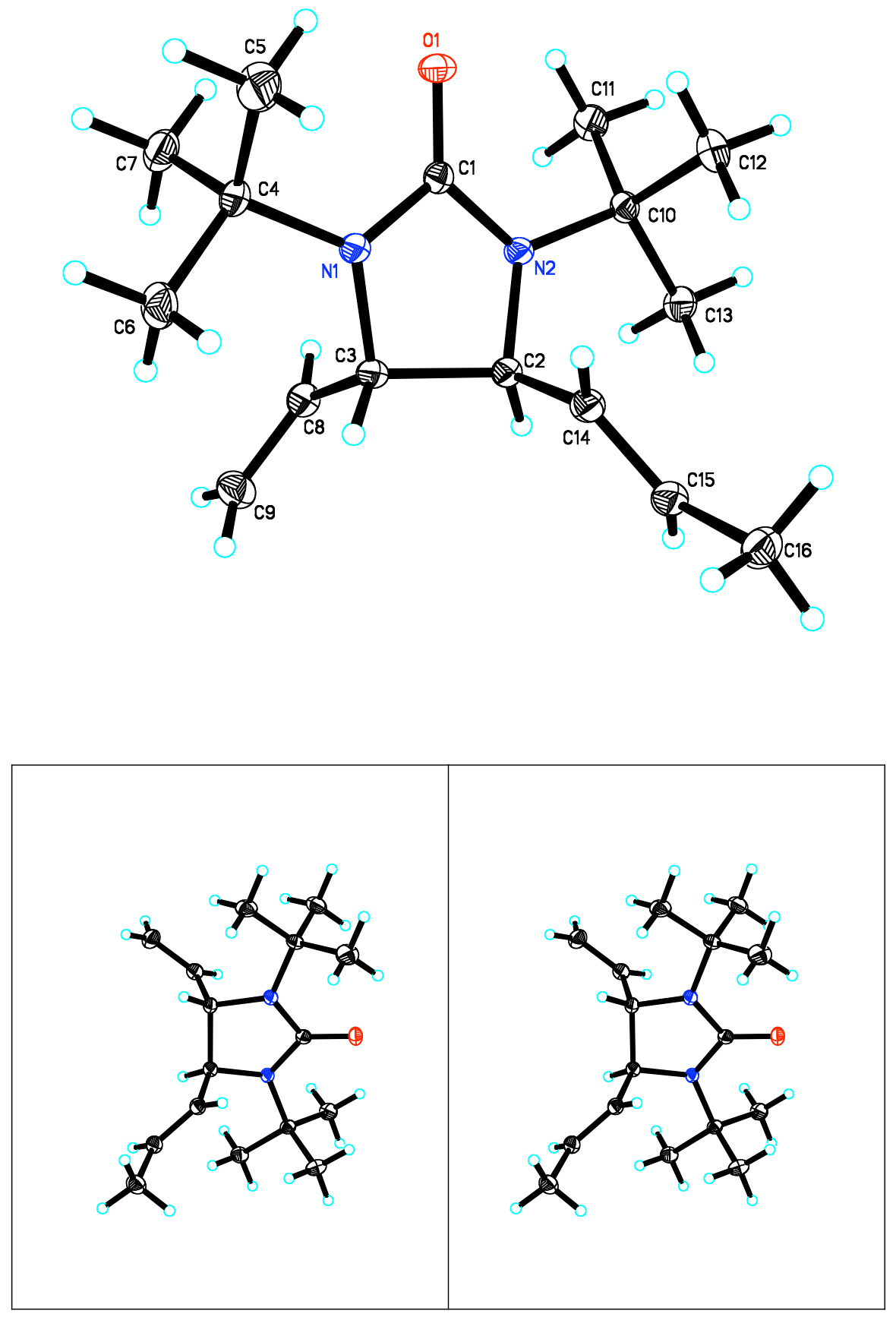
Table 1. Crystal data and structure refinement for $\mathbf{7 n}$.

Identification code

Empirical formula

Formula weight

Temperature

Wavelength

Crystal system

Space group

Unit cell dimensions

Volume

Z

Density (calculated)

Absorption coefficient

$\mathrm{F}(000)$

Crystal size

Theta range for data collection

Index ranges

Reflections collected

Independent reflections

Completeness to theta $=30.89^{\circ}$

Absorption correction

Max. and min. transmission

Refinement method

Data / restraints / parameters

Goodness-of-fit on $\mathrm{F}^{2}$

Final $\mathrm{R}$ indices [I $>2 \operatorname{sigma}(\mathrm{I})]$

$\mathrm{R}$ indices (all data)

Largest diff. peak and hole ys 153

C16 H28 N2 O

264.40

373(2) K

$0.71073 \AA$

Orthorhombic

Pbca

$\mathrm{a}=14.1771(4) \AA$ $\alpha=90^{\circ}$.

$\mathrm{b}=11.7655(3) \AA$ $\beta=90^{\circ}$.

$\mathrm{c}=18.9609(5) \AA$ $\gamma=90^{\circ}$.

3162.69(15) $\AA^{3}$

8

$1.111 \mathrm{Mg} / \mathrm{m}^{3}$

$0.069 \mathrm{~mm}^{-1}$

1168

$0.45 \times 0.29 \times 0.19 \mathrm{~mm}^{3}$

2.15 to $30.89^{\circ}$.

$-20<=\mathrm{h}<=18,-16<=\mathrm{k}<=10,-17<=1<=27$

17806

$4849[\mathrm{R}(\mathrm{int})=0.0363]$

$97.1 \%$

multi-scan

0.9873 and 0.9696

Full-matrix least-squares on $\mathrm{F}^{2}$

4849 / 0 / 172

1.071

$\mathrm{R} 1=0.0562, \mathrm{wR} 2=0.1628$

$\mathrm{R} 1=0.0752, \mathrm{wR} 2=0.1767$

0.748 and -0.754 e. $\AA^{-3}$ 
Table 2. Atomic coordinates $\left(\times 10^{4}\right)$ and equivalent isotropic displacement parameters $\left(\AA^{2} \times 10^{3}\right)$ for $\mathbf{7 n}$. $U(e q)$ is defined as one third of the trace of the orthogonalized $U^{i j}$ tensor.

\begin{tabular}{|c|c|c|c|c|}
\hline & $\mathrm{x}$ & $\mathrm{y}$ & z & $\mathrm{U}(\mathrm{eq})$ \\
\hline $\mathrm{O}(1)$ & $552(1)$ & 7796(1) & $2128(1)$ & $18(1)$ \\
\hline $\mathrm{N}(1)$ & $1286(1)$ & $7565(1)$ & $1043(1)$ & $14(1)$ \\
\hline $\mathrm{N}(2)$ & 2075(1) & $8446(1)$ & 1912(1) & $13(1)$ \\
\hline$C(1)$ & $1232(1)$ & $7920(1)$ & $1737(1)$ & $13(1)$ \\
\hline$C(2)$ & 2773(1) & $8202(1)$ & $1356(1)$ & $13(1)$ \\
\hline$C(3)$ & $2117(1)$ & $8086(1)$ & 711(1) & $14(1)$ \\
\hline$C(4)$ & $456(1)$ & $7143(1)$ & $646(1)$ & $15(1)$ \\
\hline$C(5)$ & $32(1)$ & $6124(1)$ & $1036(1)$ & $26(1)$ \\
\hline$C(6)$ & $784(1)$ & $6735(1)$ & $-80(1)$ & 21(1) \\
\hline$C(7)$ & $-287(1)$ & $8082(1)$ & $561(1)$ & $22(1)$ \\
\hline$C(8)$ & 1914(1) & $9215(1)$ & $372(1)$ & $17(1)$ \\
\hline$C(9)$ & $2144(1)$ & $9479(1)$ & $-286(1)$ & $22(1)$ \\
\hline$C(10)$ & 2331(1) & $8733(1)$ & $2651(1)$ & $13(1)$ \\
\hline $\mathrm{C}(11)$ & $1578(1)$ & $9514(1)$ & 2964(1) & $19(1)$ \\
\hline$C(12)$ & $2426(1)$ & $7655(1)$ & $3103(1)$ & $19(1)$ \\
\hline$C(13)$ & $3270(1)$ & $9375(1)$ & $2646(1)$ & $18(1)$ \\
\hline$C(14)$ & $3333(1)$ & $7132(1)$ & $1475(1)$ & $15(1)$ \\
\hline$C(15)$ & $4268(1)$ & $7100(1)$ & $1504(1)$ & $17(1)$ \\
\hline $\mathrm{C}(16)$ & $4858(1)$ & $6057(1)$ & 1592(1) & $21(1)$ \\
\hline
\end{tabular}

Table 3. Bond lengths $[\AA]$ and angles $\left[{ }^{\circ}\right]$ for $7 \mathbf{n}$.

\begin{tabular}{ll}
\hline $\mathrm{O}(1)-\mathrm{C}(1)$ & $1.2253(17)$ \\
$\mathrm{N}(1)-\mathrm{C}(1)$ & $1.3825(19)$ \\
$\mathrm{N}(1)-\mathrm{C}(3)$ & $1.4696(18)$ \\
$\mathrm{N}(1)-\mathrm{C}(4)$ & $1.4839(18)$ \\
$\mathrm{N}(2)-\mathrm{C}(1)$ & $1.3857(17)$ \\
$\mathrm{N}(2)-\mathrm{C}(2)$ & $1.4724(18)$ \\
$\mathrm{N}(2)-\mathrm{C}(10)$ & $1.4862(19)$ \\
$\mathrm{C}(2)-\mathrm{C}(14)$ & $1.5054(19)$
\end{tabular}




\begin{tabular}{|c|c|}
\hline$C(2)-C(3)$ & $1.542(2)$ \\
\hline$C(3)-C(8)$ & $1.505(2)$ \\
\hline$C(4)-C(6)$ & $1.530(2)$ \\
\hline$C(4)-C(5)$ & $1.531(2)$ \\
\hline$C(4)-C(7)$ & $1.535(2)$ \\
\hline $\mathrm{C}(8)-\mathrm{C}(9)$ & $1.325(2)$ \\
\hline$C(10)-C(11)$ & $1.530(2)$ \\
\hline$C(10)-C(13)$ & $1.531(2)$ \\
\hline$C(10)-C(12)$ & $1.538(2)$ \\
\hline$C(14)-C(15)$ & $1.3276(19$ \\
\hline$C(15)-C(16)$ & $1.495(2)$ \\
\hline $\mathrm{C}(1)-\mathrm{N}(1)-\mathrm{C}(3)$ & $109.03(11)$ \\
\hline $\mathrm{C}(1)-\mathrm{N}(1)-\mathrm{C}(4)$ & $122.73(12)$ \\
\hline $\mathrm{C}(3)-\mathrm{N}(1)-\mathrm{C}(4)$ & $123.92(12)$ \\
\hline $\mathrm{C}(1)-\mathrm{N}(2)-\mathrm{C}(2)$ & $108.77(11)$ \\
\hline $\mathrm{C}(1)-\mathrm{N}(2)-\mathrm{C}(10)$ & $122.47(12)$ \\
\hline $\mathrm{C}(2)-\mathrm{N}(2)-\mathrm{C}(10)$ & $123.66(11)$ \\
\hline $\mathrm{O}(1)-\mathrm{C}(1)-\mathrm{N}(1)$ & $125.67(13)$ \\
\hline $\mathrm{O}(1)-\mathrm{C}(1)-\mathrm{N}(2)$ & $126.02(14)$ \\
\hline $\mathrm{N}(1)-\mathrm{C}(1)-\mathrm{N}(2)$ & $108.31(12)$ \\
\hline $\mathrm{N}(2)-\mathrm{C}(2)-\mathrm{C}(14)$ & $114.21(12)$ \\
\hline $\mathrm{N}(2)-\mathrm{C}(2)-\mathrm{C}(3)$ & $100.35(10)$ \\
\hline $\mathrm{C}(14)-\mathrm{C}(2)-\mathrm{C}(3)$ & $111.25(12)$ \\
\hline $\mathrm{N}(1)-\mathrm{C}(3)-\mathrm{C}(8)$ & $113.47(12)$ \\
\hline $\mathrm{N}(1)-\mathrm{C}(3)-\mathrm{C}(2)$ & $100.37(11)$ \\
\hline $\mathrm{C}(8)-\mathrm{C}(3)-\mathrm{C}(2)$ & $112.11(12)$ \\
\hline $\mathrm{N}(1)-\mathrm{C}(4)-\mathrm{C}(6)$ & $108.71(12)$ \\
\hline $\mathrm{N}(1)-\mathrm{C}(4)-\mathrm{C}(5)$ & $109.13(13)$ \\
\hline$C(6)-C(4)-C(5)$ & 107.96(13) \\
\hline $\mathrm{N}(1)-\mathrm{C}(4)-\mathrm{C}(7)$ & $110.89(12)$ \\
\hline$C(6)-C(4)-C(7)$ & $109.90(13)$ \\
\hline$C(5)-C(4)-C(7)$ & $110.18(13)$ \\
\hline $\mathrm{C}(9)-\mathrm{C}(8)-\mathrm{C}(3)$ & $124.25(15)$ \\
\hline $\mathrm{N}(2)-\mathrm{C}(10)-\mathrm{C}(11)$ & $109.43(11)$ \\
\hline $\mathrm{N}(2)-\mathrm{C}(10)-\mathrm{C}(13)$ & $108.59(12)$ \\
\hline
\end{tabular}




\begin{tabular}{ll}
$\mathrm{C}(11)-\mathrm{C}(10)-\mathrm{C}(13)$ & $108.28(12)$ \\
$\mathrm{N}(2)-\mathrm{C}(10)-\mathrm{C}(12)$ & $111.13(11)$ \\
$\mathrm{C}(11)-\mathrm{C}(10)-\mathrm{C}(12)$ & $109.85(12)$ \\
$\mathrm{C}(13)-\mathrm{C}(10)-\mathrm{C}(12)$ & $109.51(12)$ \\
$\mathrm{C}(15)-\mathrm{C}(14)-\mathrm{C}(2)$ & $123.81(13)$ \\
$\mathrm{C}(14)-\mathrm{C}(15)-\mathrm{C}(16)$ & $125.94(14)$ \\
\hline
\end{tabular}

Table 4. Anisotropic displacement parameters $\left(\AA^{2} \times 10^{3}\right)$ for $7 \mathbf{n}$. The anisotropic displacement factor exponent takes the form: $-2 \pi^{2}\left[h^{2} a^{* 2} U^{11}+\ldots+2 h k a^{*} b^{*} U^{12}\right]$

\begin{tabular}{|c|c|c|c|c|c|c|}
\hline & $\mathrm{U}^{11}$ & $\mathrm{U}^{22}$ & $\mathrm{U}^{33}$ & $\mathrm{U}^{23}$ & $\mathrm{U}^{13}$ & $\mathrm{U}^{12}$ \\
\hline $\mathrm{O}(1)$ & $14(1)$ & $24(1)$ & $15(1)$ & $0(1)$ & $3(1)$ & $-4(1)$ \\
\hline $\mathrm{N}(1)$ & $12(1)$ & $18(1)$ & 11(1) & $-2(1)$ & $0(1)$ & $-3(1)$ \\
\hline $\mathrm{N}(2)$ & 11(1) & $17(1)$ & 11(1) & $-2(1)$ & $1(1)$ & $-2(1)$ \\
\hline $\mathrm{C}(1)$ & $13(1)$ & $14(1)$ & $13(1)$ & $0(1)$ & $-1(1)$ & $-1(1)$ \\
\hline$C(2)$ & $12(1)$ & $16(1)$ & $12(1)$ & $-1(1)$ & $0(1)$ & $-1(1)$ \\
\hline$C(3)$ & $12(1)$ & $17(1)$ & $12(1)$ & $-1(1)$ & $1(1)$ & $-1(1)$ \\
\hline$C(4)$ & $13(1)$ & $18(1)$ & $15(1)$ & $-2(1)$ & $-3(1)$ & $-3(1)$ \\
\hline$C(5)$ & $28(1)$ & $24(1)$ & $25(1)$ & $1(1)$ & $-6(1)$ & $-13(1)$ \\
\hline$C(6)$ & 21(1) & $23(1)$ & $18(1)$ & $-7(1)$ & $-4(1)$ & $0(1)$ \\
\hline$C(7)$ & $15(1)$ & $27(1)$ & $23(1)$ & $-4(1)$ & $-4(1)$ & $2(1)$ \\
\hline$C(8)$ & $16(1)$ & $18(1)$ & $17(1)$ & $0(1)$ & $-1(1)$ & $-1(1)$ \\
\hline $\mathrm{C}(9)$ & $24(1)$ & $25(1)$ & $18(1)$ & $4(1)$ & $1(1)$ & $-2(1)$ \\
\hline$C(10)$ & $14(1)$ & $15(1)$ & 11(1) & $-2(1)$ & $-1(1)$ & $0(1)$ \\
\hline $\mathrm{C}(11)$ & $19(1)$ & $20(1)$ & $17(1)$ & $-5(1)$ & $0(1)$ & $2(1)$ \\
\hline$C(12)$ & $24(1)$ & $18(1)$ & $16(1)$ & $2(1)$ & $-4(1)$ & $0(1)$ \\
\hline$C(13)$ & $17(1)$ & $21(1)$ & $16(1)$ & $-4(1)$ & $-2(1)$ & $-4(1)$ \\
\hline$C(14)$ & $14(1)$ & $17(1)$ & $13(1)$ & $-2(1)$ & $1(1)$ & $0(1)$ \\
\hline$C(15)$ & $15(1)$ & $20(1)$ & $15(1)$ & $-2(1)$ & $1(1)$ & $1(1)$ \\
\hline$C(16)$ & $16(1)$ & $24(1)$ & 21(1) & $-2(1)$ & $-1(1)$ & $4(1)$ \\
\hline
\end{tabular}


Table 5. Hydrogen coordinates ( $\left.\mathrm{x} 10^{4}\right)$ and isotropic displacement parameters $\left(\AA^{2} \times 10^{3}\right)$ for $7 \mathbf{n}$.

\begin{tabular}{|c|c|c|c|c|}
\hline & $\mathrm{x}$ & $\mathrm{y}$ & $\mathrm{z}$ & $\mathrm{U}(\mathrm{eq})$ \\
\hline $\mathrm{H}(2 \mathrm{~A})$ & 3198 & 8851 & 1295 & 16 \\
\hline $\mathrm{H}(3 \mathrm{~A})$ & 2393 & 7563 & 366 & 17 \\
\hline $\mathrm{H}(5 \mathrm{~A})$ & 502 & 5542 & 1085 & 38 \\
\hline $\mathrm{H}(5 \mathrm{~B})$ & -493 & 5832 & 773 & 38 \\
\hline $\mathrm{H}(5 \mathrm{C})$ & -178 & 6360 & 1494 & 38 \\
\hline $\mathrm{H}(6 \mathrm{~A})$ & 1251 & 6151 & -24 & 31 \\
\hline $\mathrm{H}(6 \mathrm{~B})$ & 1051 & 7361 & -336 & 31 \\
\hline $\mathrm{H}(6 \mathrm{C})$ & 255 & 6436 & -337 & 31 \\
\hline $\mathrm{H}(7 \mathrm{~A})$ & -15 & 8714 & 314 & 32 \\
\hline $\mathrm{H}(7 \mathrm{~B})$ & -496 & 8326 & 1018 & 32 \\
\hline $\mathrm{H}(7 \mathrm{C})$ & -814 & 7793 & 299 & 32 \\
\hline $\mathrm{H}(8 \mathrm{~A})$ & 1608 & 9764 & 641 & 20 \\
\hline $\mathrm{H}(9 \mathrm{~A})$ & 2450 & 8950 & -568 & 27 \\
\hline $\mathrm{H}(9 \mathrm{~B})$ & 1998 & 10194 & -464 & 27 \\
\hline $\mathrm{H}(11 \mathrm{~A})$ & 1523 & 10186 & 2680 & 28 \\
\hline $\mathrm{H}(11 \mathrm{~B})$ & 1754 & 9722 & 3436 & 28 \\
\hline $\mathrm{H}(11 \mathrm{C})$ & 983 & 9123 & 2974 & 28 \\
\hline $\mathrm{H}(12 \mathrm{~A})$ & 2901 & 7169 & 2904 & 29 \\
\hline $\mathrm{H}(12 \mathrm{~B})$ & 1834 & 7260 & 3114 & 29 \\
\hline $\mathrm{H}(12 \mathrm{C})$ & 2604 & 7862 & 3575 & 29 \\
\hline $\mathrm{H}(13 \mathrm{~A})$ & 3210 & 10049 & 2364 & 27 \\
\hline $\mathrm{H}(13 \mathrm{~B})$ & 3753 & 8896 & 2451 & 27 \\
\hline $\mathrm{H}(13 \mathrm{C})$ & 3437 & 9582 & 3119 & 27 \\
\hline $\mathrm{H}(14 \mathrm{~A})$ & 3004 & 6454 & 1533 & 18 \\
\hline $\mathrm{H}(15 \mathrm{~A})$ & 4585 & 7789 & 1464 & 20 \\
\hline $\mathrm{H}(16 \mathrm{~A})$ & 5513 & 6264 & 1594 & 31 \\
\hline $\mathrm{H}(16 \mathrm{~B})$ & 4739 & 5543 & 1210 & 31 \\
\hline $\mathrm{H}(16 \mathrm{C})$ & 4702 & 5694 & 2031 & 31 \\
\hline
\end{tabular}


X-ray structure for 9a
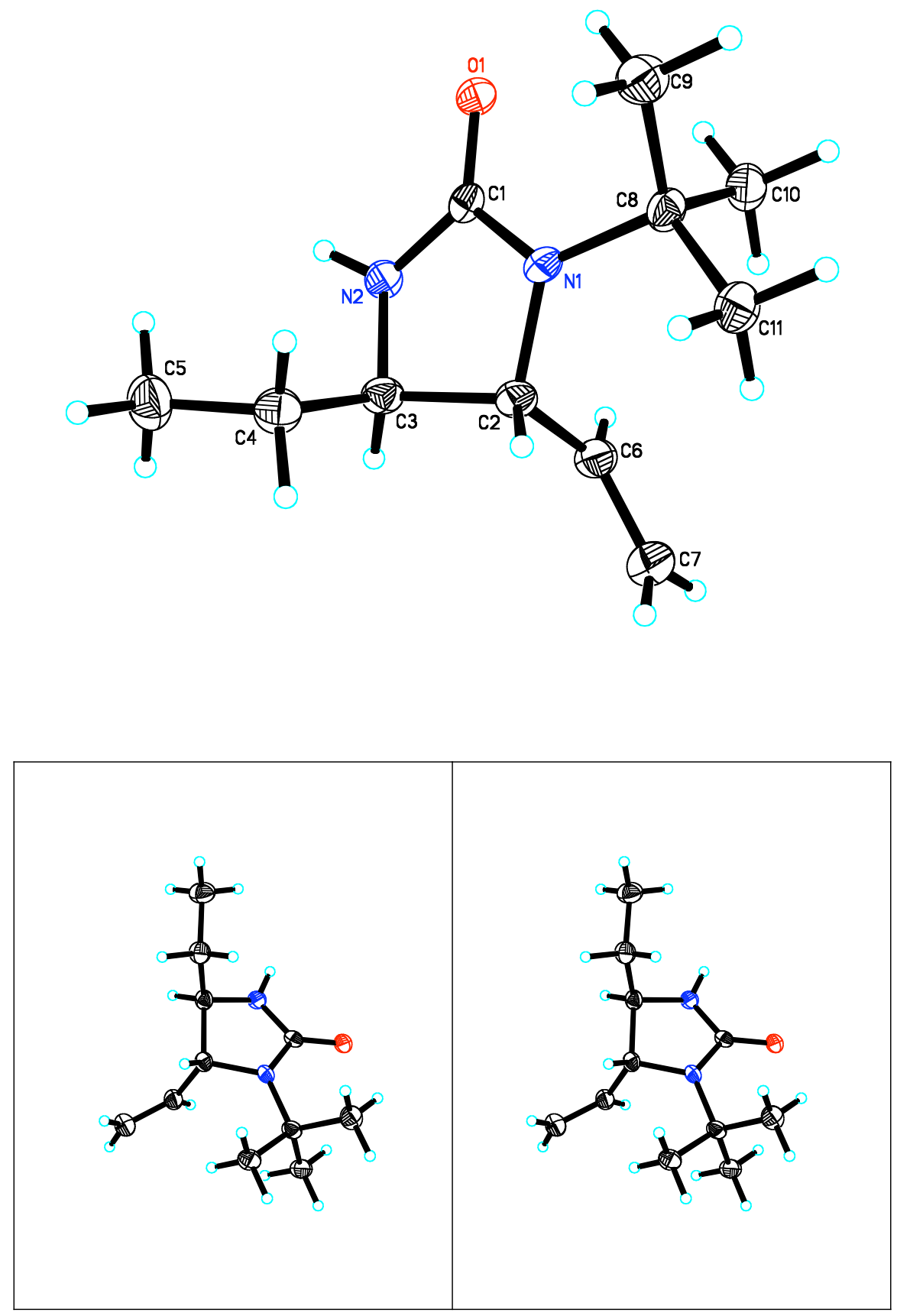
Table 1. Crystal data and structure refinement for 9a.

Identification code

Empirical formula

Formula weight

Temperature

Wavelength

Crystal system

Space group

Unit cell dimensions

Volume

Z

Density (calculated)

Absorption coefficient

$\mathrm{F}(000)$

Crystal size

Theta range for data collection

Index ranges

Reflections collected

Independent reflections

Completeness to theta $=26.37^{\circ}$

Absorption correction

Max. and min. transmission

Refinement method

Data / restraints / parameters

Goodness-of-fit on $\mathrm{F}^{2}$

Final $\mathrm{R}$ indices $[\mathrm{I}>2 \operatorname{sigma}(\mathrm{I})]$

$\mathrm{R}$ indices (all data)

Extinction coefficient

Largest diff. peak and hole ys154_0m

C11 H20 N2 O

196.29

100(2) K

$0.71073 \AA$

Triclinic

P-1

$\mathrm{a}=6.0857(7) \AA$

$\alpha=83.020(4)^{\circ}$.

$\mathrm{b}=8.7170(12) \AA$

$\beta=81.547(4)^{\circ}$.

$c=11.3604(16) \AA$

$\gamma=85.252(4)^{\circ}$.

$590.40(13) \AA^{3}$

2

$1.104 \mathrm{Mg} / \mathrm{m}^{3}$

$0.071 \mathrm{~mm}^{-1}$

216

$0.22 \times 0.17 \times 0.05 \mathrm{~mm}^{3}$

1.82 to $26.37^{\circ}$.

$-7<=\mathrm{h}<=7,-10<=\mathrm{k}<=10,-14<=\mathrm{l}<=14$

7809

$2390[\mathrm{R}(\mathrm{int})=0.0341]$

$99.2 \%$

multi-scan

0.9967 and 0.9848

Full-matrix least-squares on $\mathrm{F}^{2}$

$2390 / 0 / 132$

1.033

$\mathrm{R} 1=0.0415, \mathrm{wR} 2=0.0926$

$\mathrm{R} 1=0.0613, \mathrm{wR} 2=0.1008$

$0.018(5)$

0.266 and -0.215 e. $\AA^{-3}$ 
Table 2. Atomic coordinates $\left(\times 10^{4}\right)$ and equivalent isotropic displacement parameters $\left(\AA^{2} \times 10^{3}\right)$ for 9a. $U(e q)$ is defined as one third of the trace of the orthogonalized $U^{i j}$ tensor.

\begin{tabular}{lrrrr}
\hline & $\mathrm{x}$ & $\mathrm{y}$ & $\mathrm{z}$ & $\mathrm{U}(\mathrm{eq})$ \\
\hline $\mathrm{O}(1)$ & $5203(2)$ & $4788(1)$ & $1620(1)$ & $22(1)$ \\
$\mathrm{N}(1)$ & $3250(2)$ & $2657(1)$ & $2490(1)$ & $18(1)$ \\
$\mathrm{N}(2)$ & $4006(2)$ & $3057(2)$ & $507(1)$ & $20(1)$ \\
$\mathrm{C}(1)$ & $4237(2)$ & $3616(2)$ & $1543(1)$ & $17(1)$ \\
$\mathrm{C}(2)$ & $2686(2)$ & $1241(2)$ & $2049(1)$ & $20(1)$ \\
$\mathrm{C}(3)$ & $2484(2)$ & $1815(2)$ & $720(1)$ & $20(1)$ \\
$\mathrm{C}(4)$ & $134(2)$ & $2350(2)$ & $489(1)$ & $25(1)$ \\
$\mathrm{C}(5)$ & $-1(3)$ & $2957(2)$ & $-819(1)$ & $33(1)$ \\
$\mathrm{C}(6)$ & $4431(3)$ & $-77(2)$ & $2143(1)$ & $23(1)$ \\
$\mathrm{C}(7)$ & $4022(3)$ & $-1518(2)$ & $2564(1)$ & $32(1)$ \\
$\mathrm{C}(8)$ & $3531(2)$ & $2783(2)$ & $3752(1)$ & $20(1)$ \\
$\mathrm{C}(9)$ & $2683(3)$ & $4401(2)$ & $4064(1)$ & $28(1)$ \\
$\mathrm{C}(10)$ & $5986(3)$ & $2466(2)$ & $3921(1)$ & $26(1)$ \\
$\mathrm{C}(11)$ & $2114(3)$ & $1606(2)$ & $4573(1)$ & $26(1)$ \\
\hline
\end{tabular}

Table 3. Bond lengths $[\AA]$ and angles $\left[{ }^{\circ}\right]$ for $9 \mathbf{a}$.

\begin{tabular}{ll}
\hline $\mathrm{O}(1)-\mathrm{C}(1)$ & $1.2385(17)$ \\
$\mathrm{N}(1)-\mathrm{C}(1)$ & $1.3752(18)$ \\
$\mathrm{N}(1)-\mathrm{C}(2)$ & $1.4715(18)$ \\
$\mathrm{N}(1)-\mathrm{C}(8)$ & $1.4866(18)$ \\
$\mathrm{N}(2)-\mathrm{C}(1)$ & $1.3562(18)$ \\
$\mathrm{N}(2)-\mathrm{C}(3)$ & $1.4583(18)$ \\
$\mathrm{C}(2)-\mathrm{C}(6)$ & $1.503(2)$ \\
$\mathrm{C}(2)-\mathrm{C}(3)$ & $1.550(2)$ \\
$\mathrm{C}(3)-\mathrm{C}(4)$ & $1.517(2)$ \\
$\mathrm{C}(4)-\mathrm{C}(5)$ & $1.527(2)$ \\
$\mathrm{C}(6)-\mathrm{C}(7)$ & $1.319(2)$ \\
$\mathrm{C}(8)-\mathrm{C}(9)$ & $1.526(2)$ \\
$\mathrm{C}(8)-\mathrm{C}(11)$ & $1.531(2)$
\end{tabular}




$\begin{array}{lc}\mathrm{C}(8)-\mathrm{C}(10) & 1.532(2) \\ \mathrm{C}(1)-\mathrm{N}(1)-\mathrm{C}(2) & 109.14(11) \\ \mathrm{C}(1)-\mathrm{N}(1)-\mathrm{C}(8) & 122.55(12) \\ \mathrm{C}(2)-\mathrm{N}(1)-\mathrm{C}(8) & 124.53(11) \\ \mathrm{C}(1)-\mathrm{N}(2)-\mathrm{C}(3) & 111.33(12) \\ \mathrm{O}(1)-\mathrm{C}(1)-\mathrm{N}(2) & 125.42(12) \\ \mathrm{O}(1)-\mathrm{C}(1)-\mathrm{N}(1) & 125.73(13) \\ \mathrm{N}(2)-\mathrm{C}(1)-\mathrm{N}(1) & 108.84(12) \\ \mathrm{N}(1)-\mathrm{C}(2)-\mathrm{C}(6) & 113.80(12) \\ \mathrm{N}(1)-\mathrm{C}(2)-\mathrm{C}(3) & 101.64(11) \\ \mathrm{C}(6)-\mathrm{C}(2)-\mathrm{C}(3) & 110.93(12) \\ \mathrm{N}(2)-\mathrm{C}(3)-\mathrm{C}(4) & 113.46(12) \\ \mathrm{N}(2)-\mathrm{C}(3)-\mathrm{C}(2) & 100.33(11) \\ \mathrm{C}(4)-\mathrm{C}(3)-\mathrm{C}(2) & 114.15(12) \\ \mathrm{C}(3)-\mathrm{C}(4)-\mathrm{C}(5) & 112.95(13) \\ \mathrm{C}(7)-\mathrm{C}(6)-\mathrm{C}(2) & 124.75(15) \\ \mathrm{N}(1)-\mathrm{C}(8)-\mathrm{C}(9) & 109.08(12) \\ \mathrm{N}(1)-\mathrm{C}(8)-\mathrm{C}(11) & 108.73(12) \\ \mathrm{C}(9)-\mathrm{C}(8)-\mathrm{C}(11) & 108.14(12) \\ \mathrm{N}(1)-\mathrm{C}(8)-\mathrm{C}(10) & 110.17(12) \\ \mathrm{C}(9)-\mathrm{C}(8)-\mathrm{C}(10) & 110.56(13) \\ \mathrm{C}(11)-\mathrm{C}(8)-\mathrm{C}(10) & 110.12(12) \\ & \end{array}$

Table 4. Anisotropic displacement parameters $\left(\AA^{2} \times 10^{3}\right)$ for 9a. The anisotropic displacement factor exponent takes the form: $-2 \pi^{2}\left[h^{2} a^{* 2} U^{11}+\ldots+2 h k a^{*} b^{*} U^{12}\right]$

\begin{tabular}{lllllll}
\hline & $\mathrm{U}^{11}$ & $\mathrm{U}^{22}$ & $\mathrm{U}^{33}$ & $\mathrm{U}^{23}$ & $\mathrm{U}^{13}$ & $\mathrm{U}^{12}$ \\
\hline $\mathrm{O}(1)$ & $25(1)$ & $19(1)$ & $22(1)$ & $2(1)$ & $-4(1)$ & $-8(1)$ \\
$\mathrm{N}(1)$ & $20(1)$ & $14(1)$ & $18(1)$ & $2(1)$ & $-2(1)$ & $-4(1)$ \\
$\mathrm{N}(2)$ & $21(1)$ & $21(1)$ & $17(1)$ & $3(1)$ & $-2(1)$ & $-8(1)$ \\
$\mathrm{C}(1)$ & $15(1)$ & $17(1)$ & $19(1)$ & $2(1)$ & $-2(1)$ & $0(1)$ \\
$\mathrm{C}(2)$ & $21(1)$ & $20(1)$ & $19(1)$ & $1(1)$ & $-1(1)$ & $-8(1)$ \\
$\mathrm{C}(3)$ & $21(1)$ & $19(1)$ & $21(1)$ & $-1(1)$ & $-1(1)$ & $-6(1)$
\end{tabular}




\begin{tabular}{lllllll}
$\mathrm{C}(4)$ & $21(1)$ & $26(1)$ & $27(1)$ & $1(1)$ & $-2(1)$ & $-7(1)$ \\
$\mathrm{C}(5)$ & $27(1)$ & $39(1)$ & $33(1)$ & $1(1)$ & $-10(1)$ & $-3(1)$ \\
$\mathrm{C}(6)$ & $27(1)$ & $20(1)$ & $22(1)$ & $-2(1)$ & $-3(1)$ & $-4(1)$ \\
$\mathrm{C}(7)$ & $40(1)$ & $24(1)$ & $32(1)$ & $-1(1)$ & $-7(1)$ & $-4(1)$ \\
$\mathrm{C}(8)$ & $23(1)$ & $20(1)$ & $16(1)$ & $2(1)$ & $-1(1)$ & $-2(1)$ \\
$\mathrm{C}(9)$ & $34(1)$ & $26(1)$ & $24(1)$ & $-4(1)$ & $2(1)$ & $-1(1)$ \\
$\mathrm{C}(10)$ & $26(1)$ & $28(1)$ & $23(1)$ & $1(1)$ & $-6(1)$ & $-3(1)$ \\
$\mathrm{C}(11)$ & $31(1)$ & $28(1)$ & $18(1)$ & $2(1)$ & $0(1)$ & $-7(1)$ \\
\hline
\end{tabular}

Table 5. Hydrogen coordinates $\left(\mathrm{x} 10^{4}\right)$ and isotropic displacement parameters $\left(\AA^{2} \times 10^{3}\right)$ for 9a.

\begin{tabular}{|c|c|c|c|c|}
\hline & $\mathrm{x}$ & $\mathrm{y}$ & z & $\mathrm{U}(\mathrm{eq})$ \\
\hline HA & $4200(30)$ & $3660(20)$ & $-148(16)$ & $29(5)$ \\
\hline $\mathrm{H}(2 \mathrm{~A})$ & 1217 & 909 & 2477 & 24 \\
\hline $\mathrm{H}(3 \mathrm{~A})$ & 3078 & 976 & 209 & 24 \\
\hline $\mathrm{H}(4 \mathrm{~A})$ & -441 & 3180 & 997 & 30 \\
\hline $\mathrm{H}(4 \mathrm{~B})$ & -834 & 1474 & 724 & 30 \\
\hline $\mathrm{H}(5 \mathrm{~A})$ & -1548 & 3291 & -916 & 49 \\
\hline $\mathrm{H}(5 \mathrm{~B})$ & 526 & 2132 & -1326 & 49 \\
\hline $\mathrm{H}(5 \mathrm{C})$ & 933 & 3837 & -1054 & 49 \\
\hline $\mathrm{H}(6 \mathrm{~A})$ & 5938 & 145 & 1882 & 28 \\
\hline $\mathrm{H}(7 \mathrm{~A})$ & 2535 & -1783 & 2833 & 38 \\
\hline $\mathrm{H}(7 \mathrm{~B})$ & 5214 & -2291 & 2596 & 38 \\
\hline $\mathrm{H}(9 \mathrm{~A})$ & 1109 & 4579 & 3960 & 42 \\
\hline $\mathrm{H}(9 \mathrm{~B})$ & 3547 & 5175 & 3535 & 42 \\
\hline $\mathrm{H}(9 \mathrm{C})$ & 2850 & 4488 & 4898 & 42 \\
\hline $\mathrm{H}(10 \mathrm{~A})$ & 6499 & 1421 & 3723 & 38 \\
\hline $\mathrm{H}(10 \mathrm{~B})$ & 6151 & 2549 & 4756 & 38 \\
\hline $\mathrm{H}(10 \mathrm{C})$ & 6877 & 3226 & 3393 & 38 \\
\hline $\mathrm{H}(11 \mathrm{~A})$ & 2633 & 557 & 4387 & 39 \\
\hline $\mathrm{H}(11 \mathrm{~B})$ & 553 & 1808 & 4448 & 39 \\
\hline $\mathrm{H}(11 \mathrm{C})$ & 2249 & 1701 & 5410 & 39 \\
\hline
\end{tabular}

